\title{
Utilisation du silicium nanostructuré dans un générateur thermoélectrique pour la réduction des pertes thermiques
}

\section{Nanostructured silicon in a thermoelectric generator to reduce heat}

losses

\author{
Katir Ziouche ${ }^{1}$, Ibrahim Bel-Hadj ${ }^{1}$, Zahia Bougrioua ${ }^{1}$ \\ ${ }^{1}$ CNRS, UMR 8520-Institut d'Electronique de Microélectronique et de Nanotechnologie, Université de Lille, France, \\ e-mail: katir.ziouche@univ-lille1.fr
}

RÉSUMÉ. Dans cet article, nous présentons des microgénérateurs thermoélectriques ( $\mu$ TEG) planaires réalisés en technologie Silicium compatible CMOS, et destinés à la récupération de toute forme d'énergie thermique. Ceux-ci utilisent des matériaux faible coût, abondants et respectueux de l'environnement. La captation de la chaleur est effectuée à l'aide d'un concentrateur, en Silicium. La configuration " semi 3D » développée utilise des membranes suspendues et nécessite, pour éviter de les casser, la mise en œuvre de structures de maintien, appelées bossages. L'objectif de ce travail est d'évaluer l'amélioration des performances de conversion des $\mu$ TEG résultant de la réduction des pertes thermiques latérales suite à l'anodisation sélective de ces bossages. La simulation thermique de $\mu$ TEGs intégrant de tels bossages en Si poreux montre que les puissances générées pourront être augmentées jusqu'à $65 \%$. ABSTRACT. In this paper, we present a planar thermoelectric microgenerator ( $\mu$ TEG) based on CMOS-compatible silicon technology to harvest all forms of thermal energy. These have made by using low cost, abundant and ecofriendly materials. The heat harvesting has performed with a silicon concentrator. The developed semi-3D configuration needs safety support called 'boss' to avoid the breaking up of the membranes. The objective of this work is to evaluate the improvement of these $\mu$ TEGs performance resulting from the reduction of lateral heat losses by using selective anodizing of these bosses. The thermal modeling of $\mu$ TEGs embedding porous silicon bosses shows an increase in output power up to $65 \%$.

MOTS-CLÉS. énergie, microgénérateur, thermoélectrique, planaire, silicium poreux, modélisation.

KEYWORDS. energy, microgenerator, thermoelectric, planar, porous silicon, modelling.

\section{Nomenclature}

$\Phi$ : flux thermique [W]

$T$ : Température [K]

$\mathrm{PF}:$ Facteur de puissance $\left[\mu \mathrm{W} . \mathrm{K}^{-2} \cdot \mathrm{cm}^{-1}\right]$

A : Surface $\left[\mathrm{m}^{2}\right]$

TC : Thermocouple

IoT : Internet des objets

CC : Concentrateur de chaleur $\mathrm{e}:$ épaisseur $[\mu \mathrm{m}]$

$\alpha$ : Pouvoir thermoélectrique $\left[\mu \mathrm{V} . \mathrm{K}^{-1}\right]$

$\lambda$ : Conductivité thermique $\left[\mathrm{W} . \mathrm{K}^{-1} \cdot \mathrm{m}^{-1}\right]$

$\sigma$ : conductivité électrique [S.m ${ }^{-1}$ ]

$\Delta T_{C F}$ : différence de température entre jonctions

chaudes (C) et froides (F) de la thermopile

$\mu$ TEG : Microthermogénérateur

DC : Dissipateur de chaleur

\section{Introduction}

L'évolution du marché de l'internet des objets (IoT) a cru ces dernières années de façon quasi exponentielle avec un nombre considérable de nœuds [CIS 1]. Les IoTs offrent de très nombreuses nouvelles applications dans tous les domaines de la vie courante, allant des solutions domotiques les plus simples, passant par la santé et allant jusqu'à l'apport de solutions innovantes pour l'industrie. Néanmoins, à l'heure actuelle, la problématique majeure qui freine leur développement est 
essentiellement liée à leur alimentation électrique. Le cumul de tous les « micro-besoins » en énergie afférents va devenir colossal à l'heure où la problématique environnementale et la disparition programmée des énergies fossiles sont les principaux enjeux de demain [BRI 2], [SOU $3]$. Le nombre des $\mu$ dispositifs employés sera tel dans quelques décennies que l'utilisation des $\mu$ batteries sera proscrite tant pour l'impossibilité de les entretenir que pour leur nocivité environnementale. Produire de l'énergie, permettant l'alimentation des IoTs, à partir de $\mu$ sources collectant l'énergie fatale ou environnante, semble être la meilleure solution pour répondre à tous ces enjeux futurs. Il existe de nombreux $\mu$ dispositifs qui permettent de collecter ces énergies et les convertir en énergie électrique utile pour les IoTs [KON 4], [VUL 5], [KWO 6].

L'objet de ce travail concerne l'optimisation des performances de $\mu$ TEGs planaires réalisés en technologies Silicium CMOS dans notre laboratoire. Ceux-ci sont réalisés avec des matériaux faible coût et éco-compatibles, et sont capables de récupérer toutes les formes de chaleur. Bien que, de façon générale, le rendement de conversion énergétique des TEGs soit faible (par la nature même de la conversion thermoélectrique ou Seebeck), ce type de générateur joue un rôle important dans le domaine de la récupération d'énergie principalement en raison de l'abondance des sources de chaleur résiduelle dans l'environnement qui nous entoure [FLE 7]. Les $\mu$ TEGs présentés ici sont constitués d'une thermopile, suspendue périodiquement sur un réseau de membranes diélectriques, qui utilise l'association de longs thermocouples (TC) planaires en polysicilium/or (pSi/Au). Cette topologie résulte en des modules à résistances thermiques d'ensemble élevées [YUA 8] ; ce qui permet d'adapter thermiquement ces $\mu$ TEGs à leur environnement, conduisant à une meilleure collecte de la chaleur perdue dans l'environnement.

Plusieurs pistes d'amélioration des performances sont envisageables et sont en cours d'étude, comme par exemple l'accroissement du facteur de puissance des matériaux thermoélectriques (PF $=\alpha^{2} \sigma$, où $\alpha$ est le coefficient de conversion thermoélectrique ou coefficient Seebeck et $\sigma$ la conductivité électrique) [BOU 9]. Dans cet article, nous nous intéressons à une seconde voie d'amélioration associée à la façon de collecter la chaleur. Celle-ci est captée et canalisée dans le $\mu$ TEG au travers d'un concentrateur et d'un dissipateur qui encapsulent la thermopile suspendue ; le tout constituant de la sorte une configuration originale «semi 3D ». Nous montrons ici pour la première fois que l'utilisation de la nanostructuration sélective du Silicium constituant certaines parties du concentrateur permet de casser la thermique du système afin de privilégier le transfert de la chaleur au travers du $\mu$ TEG et ainsi optimiser la conversion thermoélectrique.

\section{Description du $\mu$ TEG}

\subsection{Principe de fonctionnement}

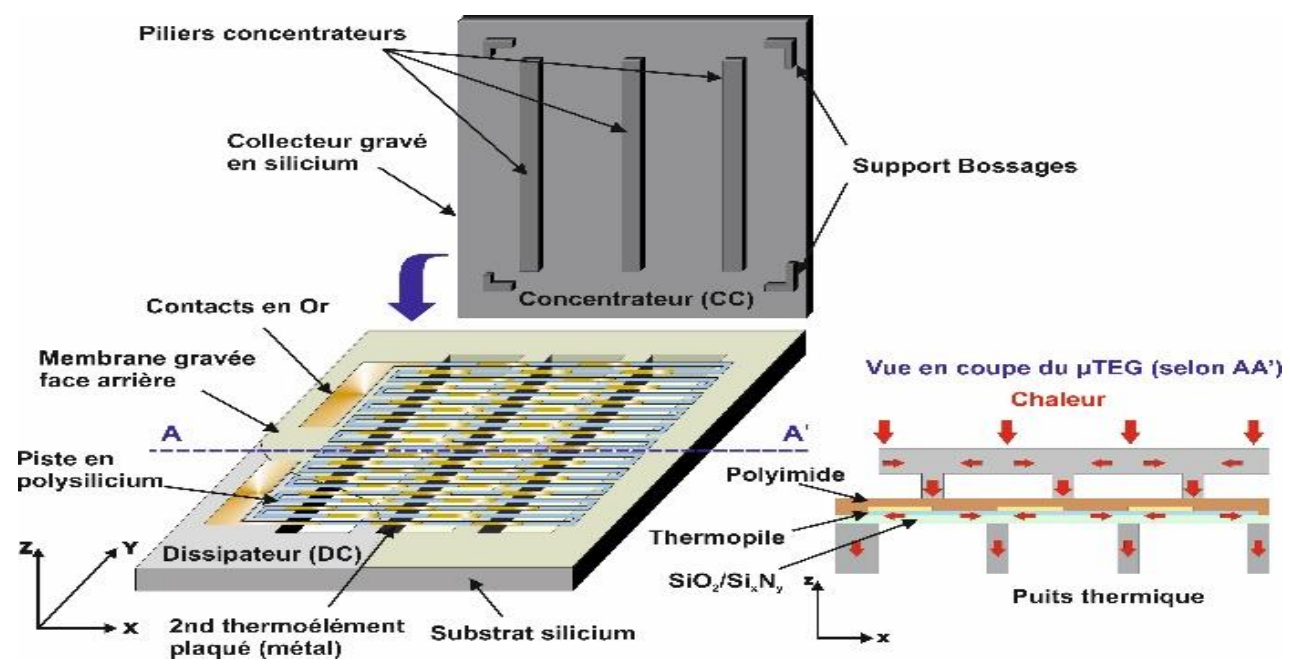

Figure 1. Structure d'un $\mu T E G$ à 3 membranes (vue «éclatée » et vue en coupe) 
La configuration de $\mu$ thermogénérateurs schématisée sur la figure 1 (surface $\sim 1 / 3 \mathrm{~cm}^{2}$ ), permet d'exploiter toute qualité d'énergie thermique [YUA 8]. Ces $\mu$ TEGs reposent sur l'utilisation d'une thermopile planaire, constituée d'un réseau de plusieurs centaines de thermocouples $\mathrm{pSi} / \mathrm{Au}$ disposés en série sur une structure de multi-membranes à contraintes mécaniques compensées (nombre de membranes pouvant varier de 2 à 10). Une telle configuration permet d'utiliser de longs TC (de 500 à $2500 \mu \mathrm{m}$ ) et ainsi d'augmenter considérablement la résistance thermique planaire entre les jonctions de ces TCs : ceci permet de maximiser les gradients thermiques entre les jonctions des TCs $\left(\Delta \mathrm{T}_{\mathrm{CF}}\right)$ et donc la tension Seebeck générée aux bornes de la thermopile $\left(\mathrm{V}_{\mathrm{S}}=\mathrm{n} \cdot \alpha_{\mathrm{TC}} \cdot \Delta \mathrm{T}_{\mathrm{CF}}\right.$, où $\mathrm{n}$ est le nombre de TCs et $\alpha_{\mathrm{TC}}$ le pouvoir thermoélectrique d'un TC). La chaleur est captée et canalisée dans un $\mu$ TEG au travers d'un concentrateur (ou collecteur, CC Figure 1), possédant autant de piliers qu'il y a de membranes, et est évacuée au travers d'un dissipateur de chaleur (DC, Figure 1). Ainsi selon la configuration du $\mu$ TEG (nombre de membranes et nombre de TCs), il est possible de générer des puissances électriques de quelques centaines de $\mu \mathrm{W} / \mathrm{cm}^{2}$ pour $1 \mathrm{~W}$ thermique injecté à travers du collecteur $\left(S_{\text {collecteur }}=0.25 \mathrm{~cm}^{2}\right)$ [BOU 9].

\subsection{Procédés de fabrication}

La réalisation des $\mu$ TEGs repose sur des techniques de fabrications utilisées dans les domaines des microtechnologies et de la microélectronique. Elle est effectuée sur des wafers Si de 3 pouces permettant la fabrication simultanée de plusieurs modules. La topologie monolithique $\mathrm{CC} /$ thermopile/membrane/DC décrite plus haut, peut être obtenue par montage simple ou par collage de deux substrats Si par technique de scellement par thermocompression. Les cavités d'air dans les deux substrats (CC et DC, Figure 1 - vue en coupe) sont réalisées par gravure ionique réactive profonde (DRIE). Elles sont disposées judicieusement pour permettre une canalisation de la chaleur captée des jonctions chauffées de la thermopile (sous les piliers du CC) vers les jonctions maintenues froides (à l'aplomb des piliers du DC). La structure de membrane suspendue est constituée par un empilement d'oxyde/nitrure/polyimide assurant la tenue mécanique et dans laquelle est encapsulée la thermopile constituée de TC en matériaux thermoélectriques (ici le polySilicium et l'or, pSi/Au mais d'autres binômes depSi/Au mais d'autres binômes de composés TE peuvent être envisagés). Le nitrure et l'oxyde de silicium (respectivement 300-500 et 800-900 nm) sont déposés par technique LPCVD (Low Pressure Chemical Vapor Deposition). Le polyimide qui permet de passiver la thermopile est déposé à la tournette et est polymérisé à $375^{\circ} \mathrm{C}$ sous $\mathrm{N} 2$. La couche de pSi est déposée par LPCVD $(\sim 600 \mathrm{~nm})$ et est gravée en forme de serpentin par gravure ionique réactive (Fig.1). La couche de métal (or) est évaporée périodiquement sur le pSi pour former les thermocouples. Chacun des TCs présente un pouvoir thermoélectrique de l'ordre de 100 à 300 $\mu \mathrm{V} / \mathrm{K}$, fonction de la nature du dopage du polySi employé.

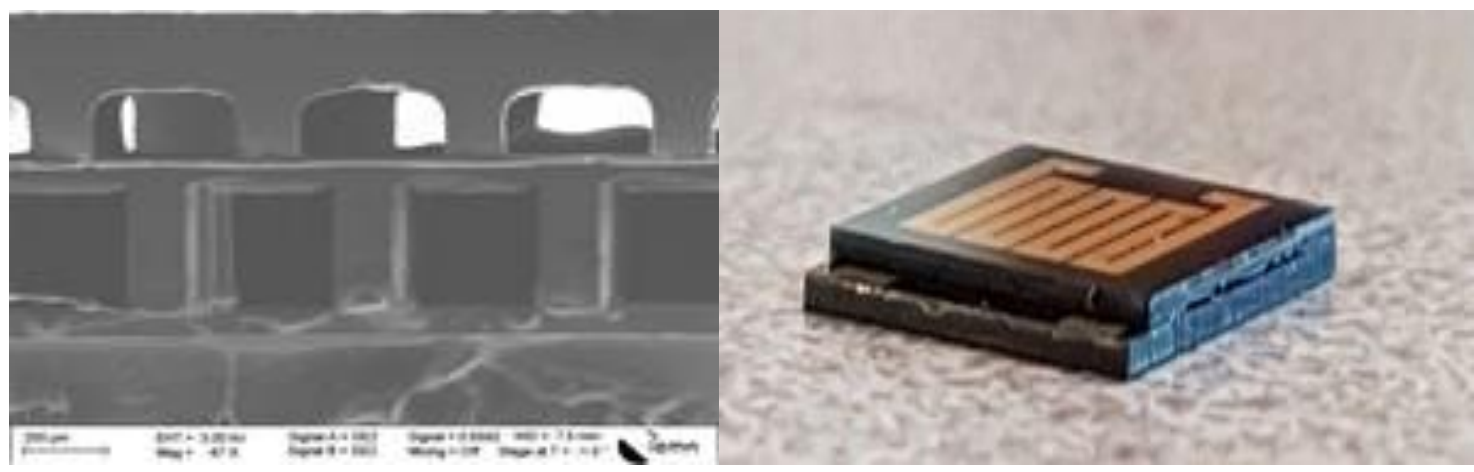

Figure 2. Vue en coupe au microscope électronique et photographie d'un $\mu T E G$ monolithe

La Figure 2 montre une image prise par microscopie électronique à balayage (MEB) dans la tranche d'un $\mu$ TEG et une photographie du module monolithe : la surface supérieure du collecteur intègre une résistance de chauffage utile pour caractériser le dispositif (imposer un flux de chaleur calibré).

(C) 2020 ISTE OpenScience - Published by ISTE Ltd. London, UK - openscience.fr 
Plusieurs configurations, de $\mu$ TEGs (surface totale identique de $5,7 \times 6 \mathrm{~mm}^{2}$ ) associant différentes longueurs de membranes, différents nombres de thermocouples et différents modes de caractérisation ont déjà fait l'objet de nos précédentes publications [YUA 8], [BOU 9], [ZIO 10], [ZIO 11], [BOU 15].

\section{Etude des transferts thermiques}

\subsection{Transfert thermique dans le $\mu T E G$}

Dans cette partie, nous décrivons les principaux transferts thermiques à travers un $\mu$ TEG lorsque celui-ci est soumis à une source de chaleur, comme indiqué sur la figure 3 . Un flux thermique de 4 $\mathrm{W} / \mathrm{cm}^{2}$ est appliqué sur la surface supérieure du concentrateur et la température de surface inférieure est fixée à $20^{\circ} \mathrm{C}$ (condition de puits thermique «parfait» à température ambiante). Les conditions de flux imposées génèrent à titre d'exemple les températures suivantes à la surface du $\mu$ TEG : $413 \mathrm{~K}$ pour la configuration à 2 membranes, $380 \mathrm{~K}$ pour la configuration à 5 membranes, et $332 \mathrm{~K}$ pour la configuration à 10 membranes.

Lorsqu'il existe une différence de température entre le concentrateur (CC) et le dissipateur (DC), le flux de chaleur total collecté et traversant le $\mu$ TEG $\phi_{\text {Total }}$ peut être décomposé en quatre principaux flux comme illustré sur la figure 3 :

$$
\phi_{\text {Total }}=\phi_{\text {Bossage }}+\phi_{\text {Convection }}+\phi_{\text {Cavité }}+\phi_{\text {Piliers }}
$$

- Le flux thermique perdu à travers les bossages situés aux quatre coins du concentrateur et qui permettent d'assurer un appui rigide du concentrateur sur le substrat (Figure 3.a) :

$$
\phi_{\text {Bossage }}=\left(\mathrm{T}_{\mathrm{CC}}-\mathrm{T}_{\mathrm{DD}}\right) \frac{\lambda_{\text {ceq }} \mathrm{A}_{\text {support }}}{\mathrm{e}_{\text {ceq }}}
$$
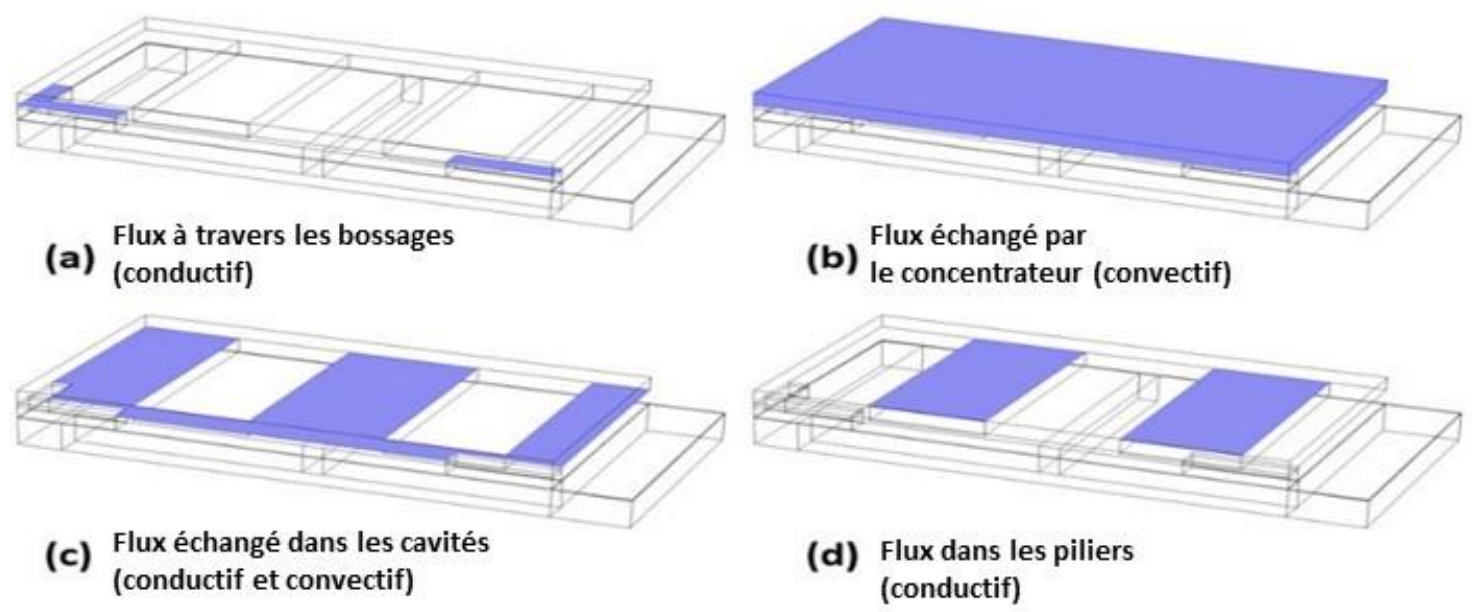

Figure 3. Principaux flux thermiques traversant un $\mu$ TEG à 2 membranes.

Seule la moitié du module est représentée

$\mathrm{A}_{\text {support }}$ est la surface totale de contact entre les bossages et le dissipateur, $\lambda_{\text {ceq }}$ et $\mathrm{e}_{\text {ceq }}$ représentent la conductivité thermique et l'épaisseur équivalente caractérisant le contact thermique entre le concentrateur et le dissipateur. Ce contact thermique est modélisé par une résistance thermique équivalent à une lame d'épaisseur $10 \mu \mathrm{m}$ et de conductivité thermique $0.063 \mathrm{~W} /\left(\mathrm{m}^{*} \mathrm{~K}\right)$ [YUA 8]. L'idéal serait d'avoir une surface $\mathrm{A}_{\text {support }}$ la plus petite possible. Cependant, une surface trop petite fragilise le $\mu$ TEG. Pour la fabrication, une surface estimée raisonnable a été fixée à $\mathrm{A}_{\text {support }}=$ $1.2 \mathrm{~mm}^{2}$. 
Le flux thermique $\phi_{\text {Convection }}$ échangé par convection entre le concentrateur et l'air ambiant (Figure 3.b) :

$\phi_{\text {Convection }}=\left(\mathrm{T}_{\mathrm{CC}}-\mathrm{T}_{\mathrm{air}}\right) \mathrm{h}_{\mathrm{air}} \mathrm{A}_{\mathrm{CC}}$

Ce flux convectif est à considérer si le $\mu$ TEG n'est pas utilisé en condition de contact avec un autre corps (i.e. récupération d'énergie radiative solaire ou autre...). $\mathrm{A}_{\mathrm{CC}}$ est la surface du concentrateur, supérieure et latérale. $\mathrm{T}_{\text {air }}$ est la température de l'air ambiant, $\mathrm{h}_{\text {air }}$ est le coefficient d'échange convectif. Ici nous considérerons une valeur conservative de $\mathrm{h}_{\text {air }}$ égale $25 \mathrm{~W} /\left(\mathrm{m}^{2} . \mathrm{K}\right)$ [ YUA 8] ; malgré cela $\phi_{\text {Convection }}$ dépasse rarement les $10 \%$.

Le flux thermique $\phi_{\text {Cavité }}$ perdu via les lames d'air emprisonnées dans les cavités creusées dans le concentrateur (flux traversant les cavités et évacué directement par le substrat sans passer à travers les membranes) qui est à la fois de type convectif et conductif.

Les calculs montrent que $\phi_{\text {Cavité }}$ est mineur $(<5 \%)$ et est d'autant plus négligeable que le nombre de cavités/membranes est élevé. Considérant les faibles épaisseurs des cavités supérieures et inférieures ( $\mathrm{e}_{\text {sup }}=200 \mu \mathrm{m}$ et $\mathrm{e}_{\text {inf }}=380 \mu \mathrm{m}$ respectivement), les coefficients d'échanges thermiques dans les cavités $h_{\text {sup }}$ et $h_{\text {inf }}$ ont été calculés avec le rapport entre la conductivité thermique de l'air et l'épaisseur de chaque cavité $: \mathrm{h}_{\text {sup }}=\lambda_{\text {air }} / \mathrm{e}_{\text {supérieure }}$ et $\mathrm{h}_{\text {inf }}=\lambda_{\text {air }} / \mathrm{e}_{\text {inf }}$. Dans ces conditions, le transfert dans les cavités est basé, en partie, sur la conduction gazeuse [YUA 8].

Le flux thermique $\phi_{\text {Piliers }}$ circulant dans les $\mathbf{M}$ piliers du concentrateur se déverse quasi intégralement le long des $\mathrm{M}$ membranes et compte tenu de la résistance thermique de chaque membrane $R_{\text {thmemb }}$ il engendre le gradient de température entre les jonctions de chaque TC de la thermopile $\Delta \mathrm{T}_{\mathrm{CF}}$.

Il est à noter que la résistance d'ensemble du $\mu$ TEG $R_{\text {thpTEG }}$ est principalement fixée par la résistance thermique des membranes.
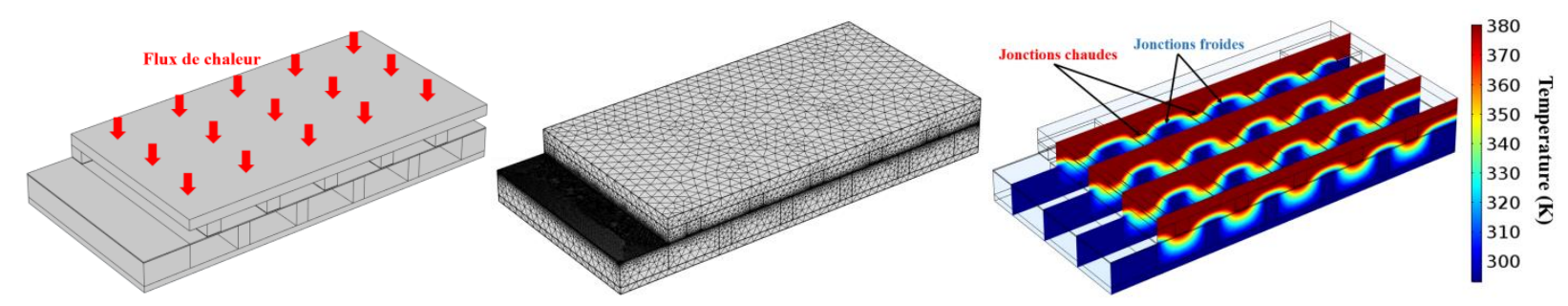

Figure 4. Structure de simulation d'un demi- $\mu$ TEG à 5 membranes et distribution de la température $\left(\right.$ COMSOL 3D $\left.{ }^{\circledR}\right)$

\subsection{Pertes thermiques aux bossages}

Nos études précédentes [YUA 12] ont montré que dans chaque $\mu$ TEG le contact direct des 4 bossages sur le dissipateur entrainait des pertes thermiques nonnégligeables du flux incident, qui ne seront pas converties en énergie électrique par la thermopile. On montre que cette quantité, $\phi_{\text {Bossages }}$, est fonction de la résistance thermique des membranes et s'accroit avec leur longueur. Ainsi, la chaleur incidente est moins ponctionnée pour les $\mu$ TEGs à 10 membranes qui présentent des résistances de membranes plus faibles que les $\mu$ TEGs à 2 membranes. Une des façons de limiter la perte $\phi_{\text {Bossages }}$ a été l'utilisation de la thermique de la couche de polyimide (matériau polymère de faible conductivité thermique $\left.\lambda_{\text {polyimide }} \sim 0.2 \mathrm{~W} / \mathrm{m} . \mathrm{K}\right)$ : située entre les lieux de contact des deux substrats Silicium (ie. les M piliers), celle-ci augmente la résistance de contact perpendiculaire entre le concentrateur et le dissipateur. Néanmoins, ce matériau polymère, déposé à la tournette sur tout le wafer par centrifugation - donc y compris sur la thermopile (Figure1) - entraine aussi une 
diminution de la résistance thermique planaire de la membrane $\mathrm{R}_{\text {thmemb }}$, et par conséquent conduit à des gradients thermiques $\Delta \mathrm{T}_{\mathrm{CF}}$ moins élevés. Selon le nombre de membranes un des deux effets antagonistes l'emporte sur l'autre.

Pour quantifier le bénéfice du polyimide, nous avons évalué les pertes thermiques à travers les bossages par simulation de la structure du $\mu$ TEG à l'aide du logiciel COMSOL 3D ${ }^{\circledR}$. Dans ce modèle, toutes les parties constituant le $\mu \mathrm{TEG}$ sont construites en 3D à partir de leurs dimensions et conductivités thermiques réelles. La structure du $\mu$ TEG étant symétrique, le calcul de la distribution de température le long de la thermopile n'est effectuée que sur la moitié du $\mu$ TEG afin d'augmenter la vitesse et la précision des calculs. Une illustration d'une structure modélisée et de la distribution de température afférente pour un $\mu$ TEG constitué de 5 membranes, est donnée figure 4.

Les Figures 5.a et 5.b montrent les évolutions obtenues par simulations numériques des gradients de températures et des flux thermiques perdus à travers les bossages en fonction de l'épaisseur de polyimide pour un flux injecté de $\phi_{\text {Total }}=1,2 \mathrm{~W}\left(4 \mathrm{~W} / \mathrm{cm}^{2}\right)$. Le flux perdu dans les cavités est tout au plus de quelques $\mathrm{mW}$.

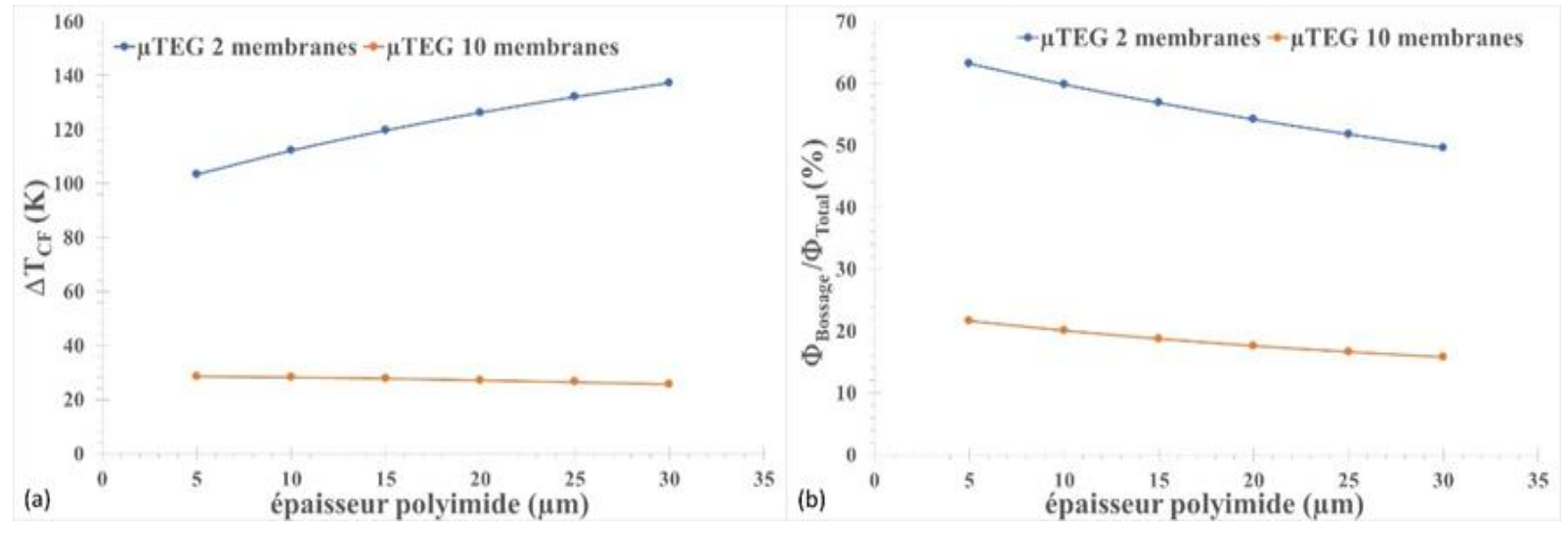

Figure 5. (a) Gradients de température $\Delta T_{C F}$ et (b)Pourcentage de flux perdu dans les bossages en fonction de l'épaisseur de polyimide

Pour les $\mu$ TEGs composés de grandes membranes $R_{\text {thmemb }}$ est plus élevée que pour ceux constitués de petites et de ce fait, à épaisseur de PI donné, $\phi_{\text {Bossages }}$ est plus important pour $\mu$ TEG à 2 membranes que pour un $\mu$ TEG à 10 membranes. On calcule que pour un $\mu$ TEG à 2 membranes, une augmentation de l'épaisseur du polyimide sera bénéfique : pour une épaisseur variant de 5 à $30 \mu \mathrm{m}$ on obtient une réduction significative de la perte thermique $\phi_{\text {Bossages }} / \phi_{\text {Total }}$ de $63,2 \%$ à $49,6 \%$, alors que la résistance thermique $\mathrm{R}_{\text {thmemb }}$ ne change pas trop. Ceci entraine une augmentation de $\Delta \mathrm{T}_{\mathrm{CF}}$ : il passe de $103 \mathrm{~K}$ à $137 \mathrm{~K}$, soit, comme la puissance électrique générée $\mathrm{P}_{\mathrm{MAX}}$ varie comme le carré de $\Delta \mathrm{T}$, une amélioration potentielle de $\mathrm{P}_{\mathrm{MAX}} \mathrm{d}$ 'un facteur 1,76 . Par contre, pour les $\mu \mathrm{TEGs}$ à petite longueur de membranes (par exemple 10, Figure5), le flux thermique perdu via les bossages a beau être plus modique, ceci ne permet pas de contrebalancer le fait que $\mathrm{R}_{\text {thmemb }}$ est davantage affectée par l'épaisseur du polyimide : ici cela résulte plûtot en une légère baisse de $\Delta \mathrm{T}_{\mathrm{CF}}$; il passe de 28,5 à $25,8 \mathrm{~K}$ lorsque l'épaisseur de polyimide augmente de 5 à $30 \mu \mathrm{m}$, d'où une réduction potentielle de $\mathrm{P}_{\text {MAX }}$ d'un facteur 0,82. Jouer avec l'épaisseur du polyimide n'est pas rentable pour toutes les configurations de module.

\section{Nanostructuration des bossages}

\subsection{Choix du silicium poreux}

L'avantage des différentes configurations de $\mu$ TEG est qu'il est possible d'adapter la résistance thermique d'ensemble du module, pour l'adapter à son environnement direct, en fixant la longueur 
des membranes. Dans la partie précédente, nous avons présenté le bénéfice lié à l'utilisation du polyimide et montré qu'il n'était valide que pour certaines configurations de $\mu$ TEGs, Par ailleurs, les techniques utilisées pour le déposer ne permettent pas des épaisseurs supérieures à $20 \mu \mathrm{m}$ et, une épaisseur trop importante peut compromettre la stabilité mécanique de l'ensemble des structures suspendues. Nous utilisons typiquement des épaisseurs de l'ordre de 15-17 $\mu \mathrm{m}$.

C'est ainsi qu'une solution supplémentaire pour diminuer davantage les pertes thermiques est actuellement à l'étude. Elle consiste à réduire les pertes latérales à travers les bossages en utilisant un procédé de gravure électrochimique appelé anodisation pour nanostructurer localement le Silicium afin de réduire la conductivité thermique de celui-ci au niveau des bossages. En effet, si on considère que les porteurs de chaleur dans les matériaux semiconducteurs sont principalement les phonons, la solution trouvée pour réduire la conductivité thermique du Silicium $\left(\lambda_{\mathrm{Si}} \sim 140 \mathrm{~W} \cdot \mathrm{m}^{-1} \cdot \mathrm{K}^{-}\right.$ ${ }^{1}$ ) est de réduire le libre parcours moyen des phonons en le nano-structurant. Cette technique efficace permet d'obtenir du silicium poreux ( $\mathrm{SiPo}$ ) dont les nanostructures solides spongieuses ou colonnaires où les nano-pores sont fortement interconnectés, confère au tout une conductivité thermique très faible $\left(\lambda_{\mathrm{SiP}} \sim 2 \mathrm{~W} \cdot \mathrm{m}^{-1} \cdot \mathrm{K}^{-1}\right)$.
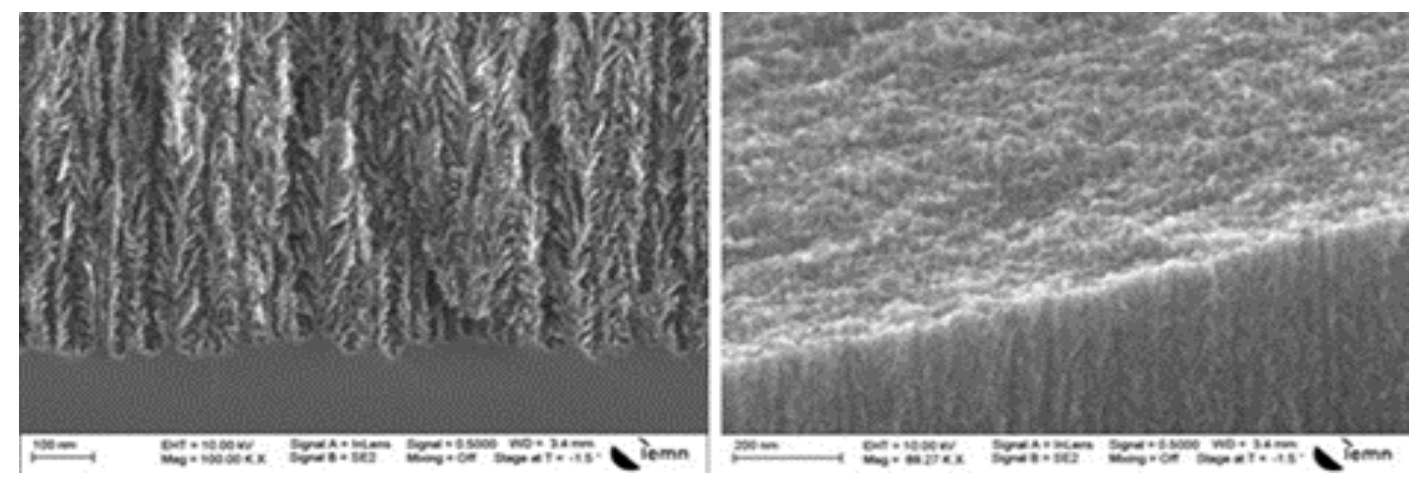

Figure 6. Photographies au MEB de la porosité dans la tranche de silicium mésoporeux

Cependant, la baisse de conductivité thermique avec la nanostructuration des bossages s'accompagne d'une fragilisation importante de la structure à ces endroits : ceci exclue la réalisation de couches trop épaisse de silicium poreux. De nombreuses études ont été nécessaires pour élaborer et stabiliser les couches de Silicium méso-poreux par traitement thermique (oxydation) et trouver un compromis intéressant entre des propriétés mécaniques autorisant la réalisation de structures épaisses et une thermique améliorée de ces couches $\left(\lambda_{\text {SiP oxydé }} \sim 1 \mathrm{~W} \cdot \mathrm{m}^{-1} \cdot \mathrm{K}^{-1}\right)$ [ZIO 13], [FAK 14].

La figure 6 montre des images MEB de couches de SiPo. Celles-ci présentent des structures spongieuses colonnaires perpendiculaires à la surface du $\mu \mathrm{TEG}$, avec une porosité de $56 \%$ et une taille de pores de 10 à $20 \mathrm{~nm}$ (correspondant à la conductivité thermique donnée précédemment), et elles sont obtenues dans un électrolyte à base d'acide fluorhydrique et d'éthanol (HF/éthanol/eau désionisée) avec une densité surfacique de courant de $100 \mathrm{~mA} / \mathrm{cm}^{2}$ (substrat $\mathrm{Si}\langle 100\rangle$ dopé Bore résistivité $\sim 0.01 \Omega . \mathrm{cm}-$ vitesse d'anodisation de $5 \mu \mathrm{m} / \mathrm{min}$ ).

\subsection{Réduction efficace des pertes}

La figure 7 illustre schématiquement une structure de $\mu$ TEG à 2 membranes avec porosification des bossages [ZIO 15]. Dans la configuration avec porosité localisée des bossages : l'augmentation considérable de leur résistance thermique permet de réduire la chaleur qui les traverse (chaleur perdue) et conduit à une meilleure canalisation du flux de chaleur incident à travers les piliers centraux en contact avec les jonctions chaudes de la thermopile. 
Les figures 8.a et 8.b donnent les résultats de simulations COMSOL 3D pour respectivement les gradients de température entre les jonctions des thermocouples et les pourcentages de flux perdus à travers les bossages pour les configurations de $\mu$ TEG à 2 et 10 membranes, et ce en fonction de l'épaisseur des bossages anodisés (poreux). Pour ce calcul l'épaisseur de polyimide est prise égale à $15 \mu \mathrm{m}$ (valeur expérimentale typique) et le flux thermique incident est de 1,2W.

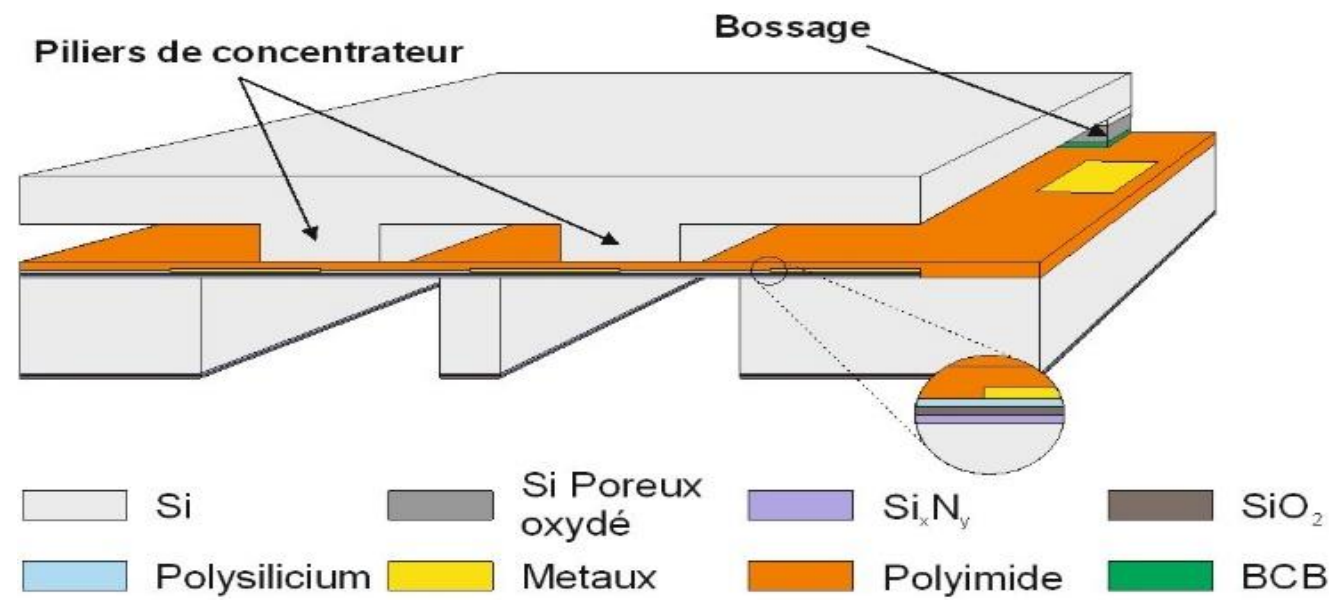

Figure 7. Illustration de la porosification des bossages dans un cas de module à 2 membranes
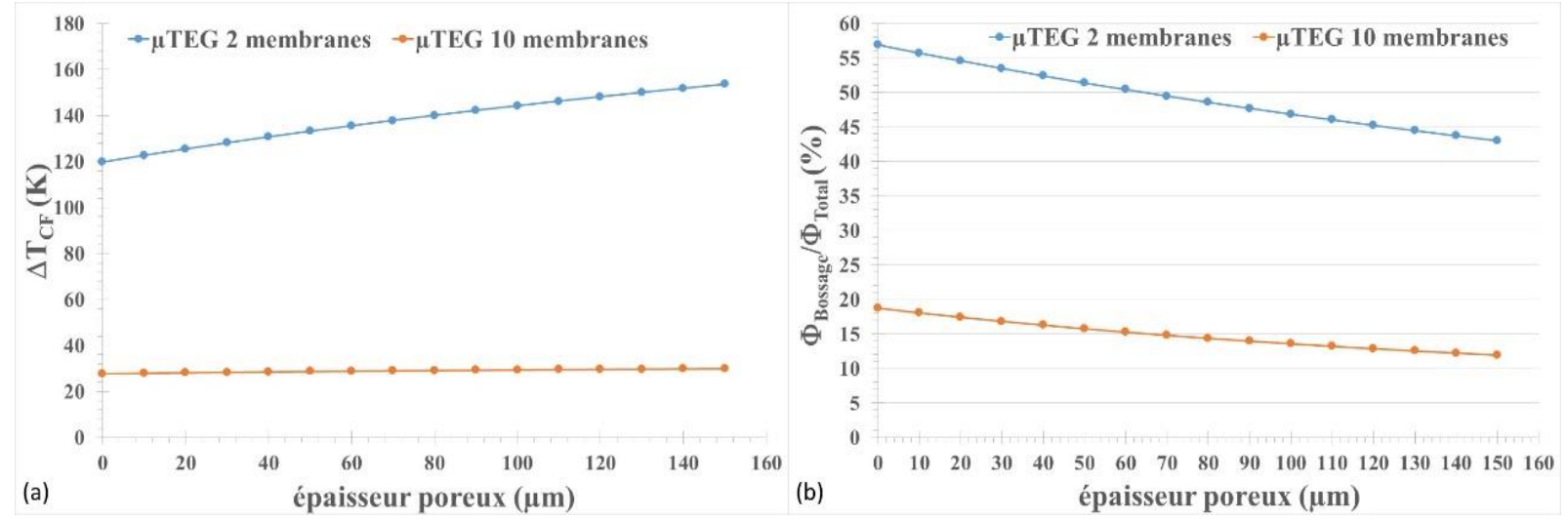

Figure 8. Gradients de température $\Delta T_{C F}$ et Flux perdu à travers les bossages porosifiés ( $\mu T E G$ à 2 et 10 membranes et densité surfacique de flux thermique incident de $4 \mathrm{~W} / \mathrm{cm}^{2}$ )

Ces résultats illustrent le fait que la performance des $\mu$ TEGs s'améliore pour des bossages de plus en plus poreux, quel que soit le nombre de membrane des modules. Les pertes dans les bossages qui sont d'environ respectivement $57 \%$ et $19 \%$ pour les $\mu$ TEGs à 2 membranes et à 10 membranes lorsque les bossages ne sont pas anodisés (non poreux), se réduisent d'un quart à un tiers, à respectivement $43 \%$ et $12 \%$ lorsqu'il y a $150 \mu \mathrm{m}$ de SiPo dans les bossages (Figure 8.b). Comme

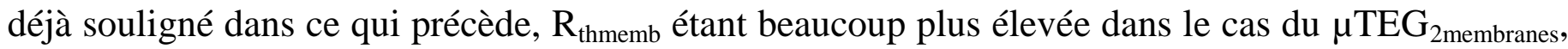
on trouve une plus grande dissipation de la chaleur à travers les bossages que dans le cas du $\mu \mathrm{TEG}_{10 \text { membranes }}$ quelle que soit l'épaisseur de bossage rendue poreuse. Au niveau des gradients thermiques tout ceci se traduit par une augmentation d'un facteur 1,28 et 1,08 respectivement pour le $\mu \mathrm{TEG}_{2 \text { membranes }}$ et le $\mu \mathrm{TEG}_{10 \text { membranes }}$ (Figure 8.a) : $\Delta \mathrm{T}_{\mathrm{CF}}$ passe de 120 à $153,6 \mathrm{~K}$ et de 27,7 à $30 \mathrm{~K}$. Ainsi la puissance de sortie générée $\mathrm{P}_{\mathrm{MAX}}$ serait, pour une même puissance incidente dans le $\mu$ TEG, augmentée de $64,4 \%$ et de $17 \%$ (resp $\mu \mathrm{TEG}_{2 \text { membranes }}$ et $\mu \mathrm{TEG}_{10 \text { membranes }}$ ).

Ainsi l'optimisation du guidage de la chaleur à travers les $\mu$ TEGs en ayant rendu les bossages poreux est plus efficace que le seul ajustement via l'épaisseur du polyimide. Cette méthode offre la possibilité d'améliorer significativement leurs performances. Ces prédictions pourront 
prochainement être corrélées au comportement expérimental de $\mu$ TEGs intégrant des bossages en silicium poreux dont la fabrication est en cours de finalisation.

\section{Conclusion}

Dans cet article, nous avons présenté des microgénérateurs thermoélectriques planaires pour la conversion d'énergie thermique en électricité. L'un des aspects essentiels dans ce type de récupération est l'amélioration de la thermique d'ensemble des dispositifs réalisés par la simulation. Les résultats obtenus sous COMSOL 3D des $\mu$ TEGs montrent qu'il est possible d'améliorer fortement leurs performances en utilisant du Silicium poreux pour réduire les pertes latérales dans les supports de maintien mécaniques. Les résultats expérimentaux à venir devraient confirmer ces prédictions. La technologie qui a été mise en œuvre intègre des matériaux respectueux de l'environnement. Les $\mu$ TEGs à rendement amélioré devraient rapidement permettre d'adresser de nouvelles applications et solutionner les problématiques d'apport en énergie électrique notamment dans les domaines des IoTs.

\section{Remerciements}

Ce travail a été soutenu par le réseau français RENATECH.

\section{Bibliographie}

[CIS 1] Cisco, Internet of Everything (IoE) Connections Counter. (https://newsroom.cisco.com/featurecontent?ArticleId=1208342), 2013.

[BRI 2] British Petroleum, 67th Statistical review of World Energy June 2018, British Petroleum, 2018. (htpps://www.bp.com/content/dam/bp/en/corporate/pdf/energy-economics/statistical-review/bp-statsreview-2018full-report.pdf).

[SOU 3] L. de Sousa, World oil and gas productions forecasts up to 2100, The Oil Drum. (//europe.theoildrum.com/node/3565).

[KON 4] Kong L.B., Li T., Hang H. H., Boey F., Zhang T., Li S., Waste Energy Harvesting, Springer Berlin Heidelberg, Berlin, Heildelberg, 2014, (//link.springer.com/10.1007/978-3-642-54634-1).

[VUL 5] Vullers R.J.M., van Schaijk R., Doms I., Van Hoof C., Mertens R., Micropower energy harvesting, SolidState Electron. 53 (2009) 684-693.

[KWO 6] Kwon D. S., Ko H. J., Kim J., Piezoelectric and electromagnetic hybrid energy harvester using two cantilevers for frequency up conversion, 2017 IEEE 30th Int. Conf. on MEMS Proceedings of, pp. 49-52.

[FLE 7] Fleurial J.P., Snyder G.J., Herman J.A., Smart M., Shakkottai P., Giauque P.H., Nicolet M.A., "Miniaturized thermoelectric power sources", 34th Intersociety Energy Conversion Engineering Conference Proceedings (1999), 1999-01-2569.

[YUA 8] Yuan Z., Ziouche K., Bougrioua Z., Godts P., Lasri T., Leclercq D., A planar micro thermoelectric generator with high thermal resistance, Sensors and Actuators A 221 (2015), p. 67-76.

[BOU 9] Bougrioua Z., Lejeune P., Leclercq D., Ziouche K., Performance of planar $\mu$ TEG as a function of polySi properties and device membrane-based topology, ECT2016-Lisbon, book of abstracts atwww.ctn.tecnico.ulisboa.pt/ect2016-conference/.

[ZIO 10] Ziouche K., Yuan Z., Lejeune P., Lasri T., Leclercq D., Bougrioua Z., Silicon-Based Monolithic Planar Micro Thermoelectric Generator Using Bonding Technology, JMEMS Letters 26 (2017), 45-47.

[ZIO 11] Ziouche K., Bougrioua Z., Yuan Z., Lejeune P., Lasri T. et Leclercq D., Microthermogénérateur thermoélectrique en technologie silicium monolithique, Actes TELECOM'2017 \& 10ième JFMMA (Rabat, 10-12 Mai 2017), pp 1-5.

[YUA 12] Yuan Z., Etude et réalisation de microgénérateurs thermoélectriques planaires en technologie silicium, thèse de doctorat, décembre 2012, Lille, France. 
[ZIO 13] Ziouche K., Bougrioua Z., Lejeune P., Lasri T., Leclercq D., Probing Technique for Localized Thermal Conductivity Measurement, Measurement Science and Technology, 26 (2015) 087003.

[FAK 14] Fakiri S., Montagne A., Rahmoun K., Iost A., Ziouche K., Mechanical properties of porous silicon and oxidized porous silicon by nanoindentation technique, Materials Science \& Engineering A 711 (2018), 470-475.

[BOU 15] Bougrioua Z., Leclercq D., Lejeune P., Ziouche K., Improvement of the performance of membrane-based planar micro thermoelectric generators as a function of polySilicon properties, to be submitted (February 2020). 